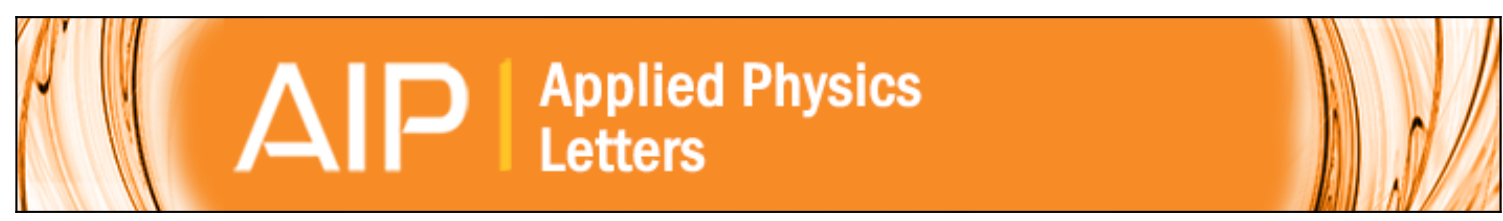

\title{
Co/Ag multilayer film: Role of annealing on the magnetic properties
}

L. F. Schelp, G. Tosin, M. Carara, M. N. Baibich, A. A. Gomes, and J. E. Schmidt

Citation: Applied Physics Letters 61, 1858 (1992); doi: 10.1063/1.108397

View online: http://dx.doi.org/10.1063/1.108397

View Table of Contents: http://scitation.aip.org/content/aip/journal/apl/61/15?ver=pdfcov

Published by the AIP Publishing

\section{Articles you may be interested in}

Ion-beam modification of Co/Ag multilayers II: Variation of structural and magnetic properties with Co layer thickness

J. Appl. Phys. 87, 8513 (2000); 10.1063/1.373571

Magnetic properties and domains in Co-Ag granular thin films

J. Appl. Phys. 87, 4837 (2000); 10.1063/1.373175

Studies of optical and electronic properties in Co/Ag multilayers

J. Appl. Phys. 81, 5256 (1997); 10.1063/1.364485

Structural and magnetic properties of Co/Ag multilayers

J. Appl. Phys. 76, 6607 (1994); 10.1063/1.358208

Magnetism and transport properties of evaporated Co/Ag multilayers

J. Appl. Phys. 73, 3910 (1993); 10.1063/1.352879

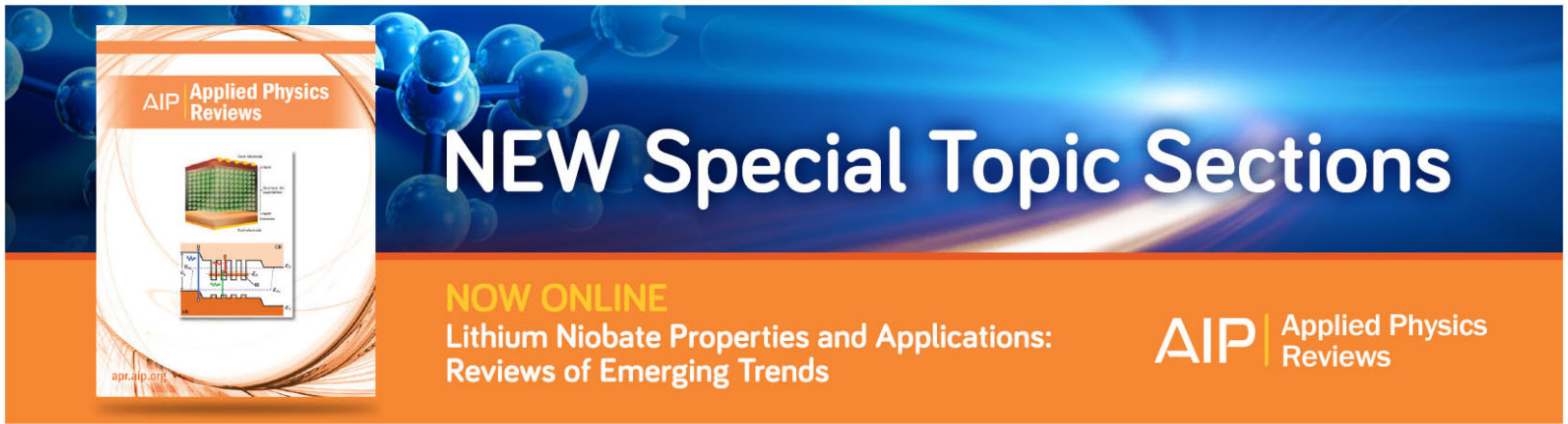




\title{
Co/Ag multilayer film: Role of annealing on the magnetic properties
}

\author{
L. F. Schelp, G. Tosin, M. Carara, M. N. Baibich, A. A. Gomes, ${ }^{\text {a) }}$ and J. E. Schmidt \\ Instituto de Fisica, Universidade Federal do Rio Grande do Sul, Rio Grande do Sul, C.P. 15051, \\ 91500-Porto Alegre, Brazil
}

(Received 26 February 1992; accepted for publication 1 August 1992)

\begin{abstract}
$\operatorname{Co}(15 \AA) / \operatorname{Ag}(60 \AA)$ multilayers produced by electron beam deposition on a $50 \AA$ chromium buffer layer over a Si(111) wafer have been studied by magnetoresistance, saturation magnetization, coercivity, and anisotropy. Annealing at various temperatures produces striking effects on the observed physical properties, such as an improvement on the value of the magnetoresistance associated to a "back-diffusion" process in the $\mathrm{Co} / \mathrm{Ag}$ interfaces. This leads one to believe that interface roughness is indeed most important to understanding the connection between giant magnetoresistance and antiferromagnetic coupling, as well as the behavior of coercivity vs interface anisotropy in these materials.
\end{abstract}

Magnetic multilayered films present new and striking magnetic properties of which the giant magnetoresistance is one of the most remarkable, as first reported by Baibich et $a .^{1}{ }^{1}$ and also observed by other workers on other systems as $\mathrm{Co} / \mathrm{Ag},{ }^{2} \mathrm{Co} / \mathrm{Cu},{ }^{3} \mathrm{Ni}_{81} \mathrm{Fe}_{19} / \mathrm{Cu} ;{ }^{4}$ its origin (antiferromagnetic coupling between adjacent magnetic layers, together with near-interface spin-dependent scattering processes) and its relation to the structural properties (interface roughness and grain size) is still a source of some controversy and several theoretical approaches have been proposed on this subject. ${ }^{5-7}$

It is well known that many physical properties are modified by annealing, which basically changes the structure of the defects, the grain size, the thickness, and/or roughness of interfaces. The observation of the corresponding effects provides further understanding of the mechanisms involved and their role as a function of the annealing temperature. These questions suggested the study of Co/ $\mathrm{Ag}$ layered structures considering that $\mathrm{Co}$ and $\mathrm{Ag}$ are immiscible to a very large extent, which should enhance interface sharpening and segregation upon thermal annealing. ${ }^{8}$ We have deposited these layered structures which are known to exhibit giant magnetoresistance and followed the evolution of their magnetic properties as a function of the annealing temperature.

The Co/Ag multilayered films were prepared by electron beam evaporation on top of a $50 \AA$ buffer layer of chromium over $\mathrm{Si}$ (111) substrates. The pressure before the evaporation was $3 \times 10^{-8}$ Torr, and the deposition

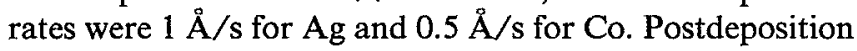
annealing was performed in a rapid thermal annealing furnace under dry hydrogen flow to ensure as close as possible to a step-wise process for the applied temperature and reduced oxidation of the samples. We have measured the saturation magnetization $\left(M_{s}\right)$, coercivity $\left(H_{c}\right)$, and the effective and perpendicular anisotropies $\left(K_{\text {eff }}, K_{\perp}\right)$ using vibrating sample and torque magnetometers. We also performed magnetoresistance measurements using a standard ac bridge method.

$\mathrm{X}$-ray diffraction patterns for as-deposited and annealed multilayers in the small angle region are shown in

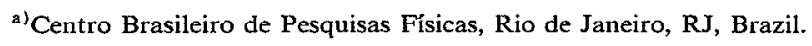

Fig. 1(a). The Bragg peaks prove the layered structure of the samples even for those annealed up to $360^{\circ} \mathrm{C}$, and above this temperature the multilayer characteristics disappear. The relative intensity of the successive peaks indicate ${ }^{\prime}$ that the samples annealed above $300^{\circ} \mathrm{C}$ indeed present lower interface width as compared to the asdeposited one. The high angle diffraction patterns, shown in Fig. 1(b), present satellites around the peak corresponding to the $\mathrm{Ag}(111)$ reflection for annealing temperatures lower than $360^{\circ} \mathrm{C}$. No significant changes can be observed in the relative intensities or broadness of the satellites probably because, as reported by Lamellas ${ }^{10}$ and coworkers, ${ }^{10}$ high angle diffaction probes local ordering, being, therefore, less sensitive to the interface quality of the sample as a whole.

As can be seen in Fig. 2(b), the saturation magnetization $M_{s}$ shows a roughly constant value up to $360^{\circ} \mathrm{C}$, indicating the overall maintenance of the multilayer characteristics confirmed by $\mathrm{x}$-ray measurements. For temperatures higher than $360^{\circ} \mathrm{C} M_{s}$ starts to decrease as a result of the degradation of the multilayered structure. The saturation magnetization involves, in an averaged way, the sum of several contributions, with different scales, so that it is less sensitive to local changes as compared to any of the other quantities studied. We have observed that all other properties show sharp modifications in the range of 260 $360^{\circ} \mathrm{C}$. Figure 2 (a) shows the effect of the annealing temperature on the resistivity, which suggests that a process associated to the reduction of the interface roughness and/ or grain growth is taking place. The electrical resistivity of metal multilayers reflects the character of their interfaces: those with higher degrees of mixing should have much higher resistivity. Therefore, since the resistivity in Fig. $2(a)$ is lowered as a function of the annealing temperature, we expect this to be related to sharper interfaces.

The resistivity measurement by itself is not conclusive, but we can see that there is an increase in the absolute value of the perpendicular anisotropy $K_{\perp}$ [Fig. 2(c)] which is proportional to $K_{v}$ (magnetocrystalline anisotropy), to $2 K_{s} /$ thickness (surface/interface anisotropy) and to $k_{\text {st }}$ (stress anisotropy). If one assumes that the magnetocrystalline and the stress contributions are less important than the interface term, the data are an indication that the in- 


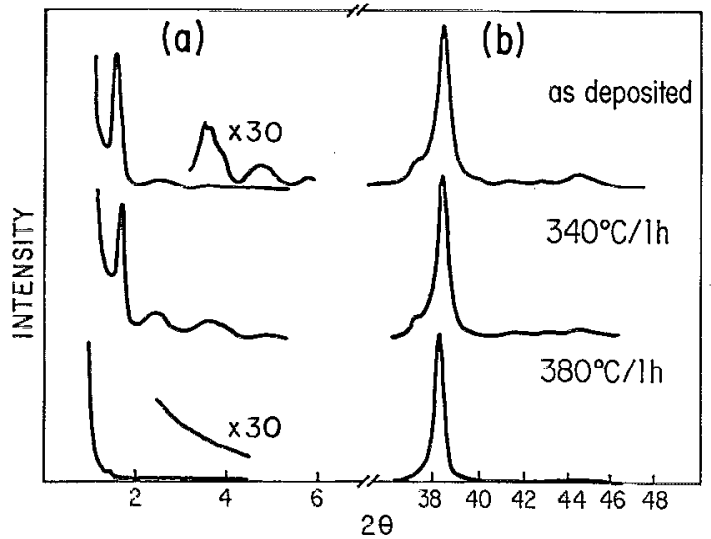

FIG. 1. X-ray diffraction patterns of as-deposited and annealed at 340 and $380^{\circ} \mathrm{C} \mathrm{Co} / \mathrm{Ag}$ multilayer for (a) small angle and (b) high angle regions.

terface sharpens as a function of the increasing annealing temperature. This sharpening is consistent with the increase of the interface anisotropy, for a given spin-orbit coupling strength, as suggested by the simple calculation of Bruno. ${ }^{11}$ On the other hand, as observed in item (d) of Fig. 2 , the coercivity $H_{c}$ increases up to $360^{\circ} \mathrm{C}$ (and beyond). This is also consistent with a sharper interface if one assumes (as claimed by Carcia et al. ${ }^{12}$ ), that coercivity and anisotropy energy are related, that is, $H_{c}$ and $K_{s} / M_{s}$ are proportional. Since $M_{s}$ is roughly constant in the annealing range where the multilayer is preserved, we can compare

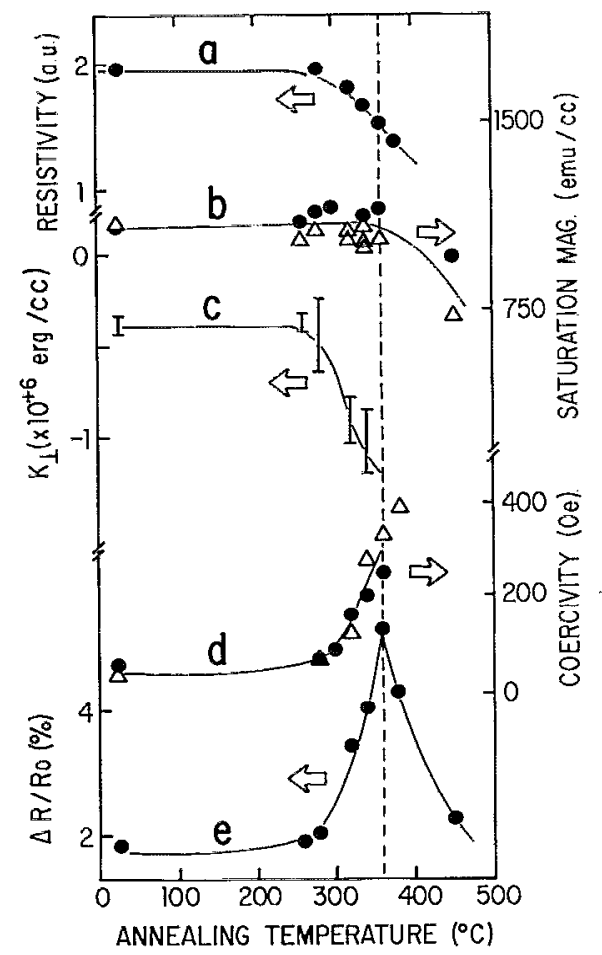

FIG. 2. Electrical resistivity (a), saturation magnetization measured by vibration sample $(O)$ and torque $(\Delta)$ magnetometers $(b)$, perpendicular anisotropy constant (c), coercive field (d), and magnetoresistance (e) as a function of annealing temperature $(1 \mathrm{~h})$. the observed ratio variation of the quantities $H_{c}\left(360^{\circ} \mathrm{C}\right) /$ $H_{c}\left(25^{\circ} \mathrm{C}\right)=5$ and $K_{1}\left(360^{\circ} \mathrm{C}\right) / K_{1}\left(25^{\circ} \mathrm{C}\right)=3$. This difference in the ratios suggests the existence of at least two contributions for the coercivity: one associated to the increased interface sharpness and a remaining one due to other factors such as grain growth and so on.

A better and clearer picture comes out when analyzing item (e) of Fig. 2, that depicts the magnetoresistance, measured at room temperature in the in-plane configuration: the observed values (varying from $2 \%$ to about $5 \%$ ) are clear indications that we are in presence of regions of the sample that couple antiferromagnetically, and with the right order of magnitude for the roughness, leading to the well-known giant magnetoresistance (GMR). The increase in the magnetoresistance effect could be accounted for by the decrease in resistivity, but it can never explain how the effect reduces for annealing temperatures above $360^{\circ} \mathrm{C}$. The only explanation left for the increase of the GMR is that, on having $\mathrm{Co}$ and $\mathrm{Ag}$ immiscible over a large temperature range, one is inducing a back-diffusion process upon annealing at low temperatures, thus effectively obtaining smoother interfaces. Other parallel effects such as grain growth and strain relief that certainly occur, are not expected to account for the observed effects. Our picture is the following: the overall antiferromagnetic characteristics of the sample grows once the back diffusion takes place; this assertion can be made from the observed increase in the antiferromagnetic coupling constant $J_{\mathrm{AF}}$, estimated ${ }^{13}$ from measured values of the saturation fields. The behavior of the magnetoresistance follows quite well the idea of having the adequate competition between interface roughness and the strength of the magnetic coupling.

Work in this problem is by no means definitive, and we are presently performing similar experiments on multilayers with different thicknesses and deposited over different buffer layers to study more closely the effects of different degrees of starting roughness, as well as the influence of both the spacer and active layers. The results of this study are to be published shortly.

In summary, we have been able to observe the effect of the interface roughness on the magnetic properties of $\mathrm{Co}(15 \AA) / \operatorname{Ag}(60 \AA)$ multilayers by inducing a backdiffusion process by means of low temperature anneals. The striking effects observed lead us to the conclusion that the interface roughness is indeed most important in defining the strength of the coupling between adjacent magnetic layers/transport properties and in determining the behavior of the coercivity and/or interface anisotropy.

We would like to thank Professor W. H. Schreiner for his invaluable help in preparing the samples. This work was supported by Conselho Nacional de Desenvolvimento Cientifico e Tecnologico ( $\mathrm{CNPq}$ ), Financiadora de Estudos e Projetos (FINEP), and Fundação de Amparo a Pesquisa (FAPERGS), Brazil.

${ }^{1}$ M. N. Baibich, J. M. Broto, A. Fert, F. Nguyen Van Dau, F. Petroff, P. Etienne, G. Creuzet, A. Friederich, and J. Chazelas, Phys. Rev. Lett. 61, 2472 (1988).

${ }^{2}$ S. Araki, K. Yasui, and Y. Nakumiya, J. Phys. Snc. Jpn. 60, 2827 (1991). 
${ }^{3}$ D. H. Mosca, A. Barthélémy, F. Petroff, A. Fert, P. A. Schroeder, W. P. Pratt, Jr., R. Laloee, and R. Cabanel, J. Magn. Magn. Mater. 93, 480 (1991).

${ }^{4}$ S. S. P. Parkin, Appl. Phys. Lett. 60, 512 (1992).

${ }^{5}$ B. L. Johnson and R. E. Camley, Phys. Rev. B 44, 9997 (1991).

${ }^{6}$ A. Barthélémy and A. Fert, Phys. Rev. B 43, 13124 (1990).

${ }^{7}$ P. M. Levy, S. Zhang, and A. Fert, Phys. Rev. Lett. 65, 1643 (1990).

${ }^{8}$ A. Yamaguchi, H. Miyashita, T. Kamemura, Y. Togawa, and R. Yamamoto, Coll. Phys. C1 51, 927 (1990).
'Y. Sasanuma, M. Uchida, K. Okada, K. Yamamoto, Y. Kitano and A. Ishitani, Thin Films 203, 113 (1991).

${ }^{10}$ T. L. Lamellas, II. David He, and R. Clark, Phys. Rev. B 43, 12296 (1991).

I'P. Bruno, Phys. Rev. B 38, 865 (1989).

${ }^{12}$ P. F. Carcia, S. I. Shah, and W. B. Zeper, Appl. Phys. Lett. 56, 2345 (1990).

${ }^{13}$ F. Nguyen-Van-Dau, thesis, Université de Paris-Sud, Orsay, France, 1989 (unpublished).

Published without author corrections 